\title{
POR UMA CIÊNCIA DOS RISCOS E VULNERABILIDADES NA GEOGRAFIA
}

\author{
a science of the risks and vulnerabilities to geography
}

Lutiane Queiroz de Almeida*

\begin{abstract}
Resumo
A humanidade passou nos últimos 50 anos por intensas mudanças na sua estrutura social, econômica, cultural e ambiental. A principal marca evolutiva de nossa sociedade é o amplo processo de urbanização. Essa ebulição cultural e espacial incitou uma série de ansiedades, medos, inseguranças. Ulrich Beck denominou a sociedade pós-moderna de a "sociedade do risco", dada a generalização de riscos diversos, perigos, vulnerabilidades, exposições, incertezas, inseguranças e medos, que são elementos típicos da sociedade atual, que é também urbana, complexa e caracterizada pelas múltiplas espacialidades. O principal objetivo deste artigo é contribuir para uma discussão teórico-conceitual sobre a noção de risco e de vulnerabilidade e, notadamente, o estabelecimento de uma Geografia dos riscos e das vulnerabilidades sociais e ambientais, com ênfase no ambiente das cidades. Para isso, fez-se uma análise de alguns dos principais referenciais teórico-metodológicos sobre a ciência dos riscos e vulnerabilidades, contribuindo com alternativas de operacionalização desses conceitos.
\end{abstract}

Palavras-chave: Risco, Vulnerabilidade, Geografia, Urbanização, Pós-Modernidade.

\begin{abstract}
The humanity passed in last the 50 years for intense changes in its social, economic, cultural and environmental structure. The main evolutive mark of our society is the ample process of urbanization. This cultural and space boiling has stirred up a series of anxieties, fears, unreliabilities. Ulrich Beck called after modern a "risk society", given the generalization of diverse risks, hazards, vulnerabilities, expositions, uncertainties unreliabilities and fears, which are typical elements of the current society, that is also urban, complex and characterized by the multiple spaceability. The main purpose of this article is to contribute for a theoretician-conceptual quarrel on the vulnerability and risk notion and, mainly, the establishment of a Geography of the risks and of the social and environmental vulnerabilities, with emphasis on the environment of the cities. For this, we carried out an analysis of some of the main theoretical and methodological references on the science of the risks and vulnerabilities, contributing with alternatives of operacionalization of these concepts.
\end{abstract}

Key words: Vulnerability, Geography, Urbanization, After Modernity.

\section{Résumé}

L'humanité est passée dans les dernière 50 années par d'intenses changements dans sa structure sociale, économique, culturelle et environnementale. La principale marque évolutive de notre société est l'ample processus d'urbanisation. Cette ébullition culturelle et spatiale a incité une série d'anxiétés, de peurs, d'insécurités. Ulrich Beck a qualifié la société postmoderne de "société de risque", étant donnée la généralisation de risques divers, dangers, vulnérabilités, expositions, incertitudes, insécurités et peurs, qui sont des éléments typiques de la société actuelle, laquelle est aussi urbaine, complexe et caractérisée par multiples spacialités. Le principal objectif de cet article est de contribuer à une discussion théorique et conceptuelle sur la notion de risque et de vulnérabilité et, principalement, à l'établissement d'une Géographie des risques et des vulnérabilités sociales et environnementales, avec un accent sur l'environnement des villes. Pour cela, il est proposé une analyse de certains des principaux référentiels théoriques et méthodologiques de la science des risques et des vulnérabilités, en contribuant à des alternatives de mise en oeuvre de ces concepts.

Mots clé: Risque, Vulnérabilité, Géographie, Urbanisation, Postmodernité.

(*) Prof. Dr. do Programa de Pós-Graduação em Geografia da Universidade Federal do Rio Grande do Norte - Campus Universitário, BR 101, Lagoa Nova, Natal, RN, CEP 59072-970., Tel: (+ 55 84) 32153571 - lutianealmeida@hotmail.com 


\section{INTRODUÇÃO}

É incontestável a onipresença do risco. Desde o momento em que a vida humana é concebida, correm-se diversos riscos. O risco é, assim, inerente à vida.

Com a presença humana, o risco não deixa de existir (não existe "risco zero"), apenas varia no tempo e no espaço. É, no entanto, principalmente na Modernidade que essa onipresença se exacerba. Entre as marcas da evolução humana recente - o surgimento do capitalismo, o progresso das ciências e das tecnologias, a disseminação das relações democráticas - a ideia revolucionária que define o limite entre a Modernidade e o passado é a busca do "domínio" do risco, ou seja, a noção de que o futuro é mais do que uma fantasia dos deuses e de que o homem não é um ser passivo diante da Natureza (BERNSTEIN, 1998).

Até o momento das Revoluções (Científica, Industrial, Francesa, Renascimento, entre outras) e durante muito tempo, as catástrofes, fossem naturais, fossem provocadas pelo homem, eram tidas como "atos de Deus".

Esse pretenso "domínio" do futuro, no entanto, parece paradoxal em razão de grandes transformações relativamente recentes na sociedade e na Natureza em âmbito planetário.

De um lado, já se aproxima da metade da população humana mundial o contingente de pessoas concentradas nos exíguos espaços das cidades, o que denota uma tendência à generalização dos riscos e perigos, já que estes aumentam com a concentração populacional e o crescimento demográfico em áreas urbanas ambientalmente instáveis e socialmente vulneráveis .

Por outro lado, as mudanças ambientais globais (aquecimento global, efeito estufa, aumento do nível dos oceanos, aumento da freqüência de fenômenos climáticos extremos, entre outros) impõem ampla vulnerabilização e um crescente sentimento de insegurança em toda a humanidade.

Para muitos autores, esses fatos - juntamente com riscos e incertezas a eles associados - são considerados como a tônica do que se convencionou chamar de Modernidade, ou ainda de Pós-Modernidade.

Para Giddens (2002), fala-se em Modernidade ao referir-se às instituições e modos de comportamento engendrados inicialmente na Europa com o fim do feudalismo, e que ao longo do século XX se tornaram mundiais em sua influência; a Modernidade se refere ao "mundo industrializado" e às relações sociais implicadas no uso generalizado das técnicas e das máquinas nos processos de produção.

O século XX, como concebe Ulrich Beck (1998) em sua clássica obra Risikogesellschaft (A sociedade do risco), não foi pobre em catástrofes históricas. Nesse momento, generalizam-se os chamados perigos tecnológicos (technological hazards) - Hiroshima e Nagasaki (Japão, 1945), Minamata (Japão, 1956), Seveso (Itália, 1976), Three Miles Island (EUA, 1979), Bophal (Índia, 1984), Chernobyl (Ucrânia, 1986), Goiânia (Brasil, 1987).

Quanto aos os perigos naturais (natural hazards), não menos importantes e não menos catastróficos, se repetem com freqüência e magnitudes cada vez mais devastadoras, tanto em função das mudanças ambientais empreendidas pelo homem, quanto pela crescente concentração de populações cada vez mais vulneráveis nas cidades, pelo crescimento demográfico, e pela globalização das desigualdades e segregação sociais.

O tsunami ocorrido no oceano Índico, em dezembro de 2004, causou milhares de mortes, destruiu milhares de habitações e levou o caos e a destruição a incontáveis famílias da Ásia e da África, principalmente populações socialmente vulneráveis. Da mesma forma, as consequências do furacão Katrina atingiram com maior gravidade as comunidades negras e pobres de Nova Orleans, em 2005.

Assim, pode-se dizer que os riscos e catástrofes são próprios da Modernidade. Ao mesmo tempo, presencia-se um momento histórico em que toda a humanidade se sente vulnerável. Até mesmo a potência econômica e bélica hegemônica mundial sentiu-se vulnerável diante dos ataques terroristas ao World Trade Center, em Nova Iorque e ao Pentágono, em Washington, em 11 de setembro de 2001 . 
Nesse contexto, o principal objetivo deste artigo é contribuir para uma discussão teórico-conceitual sobre a noção de risco e de vulnerabilidade e, principalmente, o estabelecimento de uma ciência dos riscos e das vulnerabilidades na Geografia brasileira.

\section{EVOLUÇÃO CONCEITUAL E DIMENSÃO HISTÓRICA DA ABORDAGEM DO RISCO}

A noção de risco permeia diversas nuanças da sociedade, desde a academia até o âmbito empresarial. É objeto de uso na economia (análise do risco-país, risco de queda nas bolsas de valores), na engenharia (avaliação de riscos de acidentes em construções, na segurança do trabalho), nos seguros, na saúde, ou seja, é um conceito consideravelmente difundido, por ser, entre outros motivos, o risco um componente recorrente da sociedade moderna.

A complexidade deste conceito advém, essencialmente, por ser também essa característica inerente à sociedade contemporânea permeada pela incerteza, pelo medo e pela insegurança.

O termo risco está freqüentemente acompanhado de um adjetivo, que o qualifica e que o associa ao cotidiano dessa sociedade: risco ambiental, risco tecnológico, risco natural, risco social, risco biológico, risco econômico, entre outros (cf. CASTRO et al., 2005).

Em virtude dessa diversidade teórico-conceitual da abordagem do risco, são comuns as imprecisões, ambiguidades e até confusões quanto ao estabelecimento dos conceitos. Por isso, propõe-se uma análise dos conceitos básicos, com o intuito precípuo de uma legítima precisão semântica desta abordagem.

\section{A ETIMOLOGIA E O TERMO “RISCO”}

A origem do termo "risco" é considerada por alguns autores como incerta, mas está presente em todas as línguas europeias: risk (inglês), rischio (italiano), riesgo (espanhol), risque (francês). Alguns linguistas relacionam o referido termo ao castelhano antigo "resegue" (ressecar, cortar), cuja acepção mais utilizada na Idade Média é sinônimo de luta, contradição e divisão (ANEAS DE CASTRO, 2000).

Já de acordo com a Oficina da Coordenação para o Socorro em caso de Desastres, da UNDRO (Unided Nations Disaster Relief Organization), que contribui para a definição de vários conceitos relativos ao estudo dos desastres naturais, risco corresponde ao "grau de perda previsto devido a um fenômeno natural determinado e em função tanto do perigo natural quanto da vulnerabilidade." (ANEAS DE CASTRO, 2000, p. 02).

Pode-se notar um viés específico no conceito anterior, principalmente ao definir o tipo de risco (risco natural - a ser discutido mais adiante). De forma geral, porém, o conceito de risco pode ser tomado como categoria de análise associada às noções de incerteza , exposição ao perigo, perda e prejuízos materiais e humanos, atrelados não só a processos naturais, mas também a processos oriundos das atividades humanas. De uma forma ampla, o risco "refere-se à probabilidade de ocorrência de processos no tempo e no espaço, não constantes e não determinados, e à maneira como estes processos afetam (direta ou indiretamente) a vida humana." (CASTRO et al., 2005, p. 12).

\section{O USO DA CATEGORIA RISCO}

Mesmo sendo uma categoria de análise claramente relacionada ao desenvolvimento industrial e ao uso da ciência como formas de "controle" das incertezas, a noção de risco, sua origem, análise e gestão têm sido relatadas por diversos autores, a partir da consideração da Babilônia antiga, no vale do Tigre-Eufrates (3.200 a.C.) como marco espaciotemporal do início da analise do risco (CUTTER, 1993; CASTRO et al., 2005).

Perigos ligados ao envenenamento por chumbo são relatados há séculos, e descritos na Bíblia e no Talmude. Vinhos fabricados e estocados em barris de chumbo foram considerados os responsáveis pela queda do Império Romano (CUTTER, 1993). 
As origens do conceito de risco também remontam, na pré-Modernidade, ao início das navegações, das atividades militares e das práticas mercantis. Para a maior parte das cidades pré-modernas, os incêndios e as epidemias se caracterizaram como as duas principais calamidades urbanas durante séculos, atrelados à precária existência dos citadinos da época.

Além disso, dadas as modestas dimensões das cidades antigas, as grandes catástrofes geofísicas (terremotos, erupções vulcânicas etc.) e hidrológicas (inundações, tempestades etc.) provocavam perdas e prejuízos contingenciais e limitados, se comparados aos incêndios que assolaram cidades como Toulouse - 1442 e 1551, Berlin - 1405, Amsterdam - 1451 e 1452, Moscou - 1626, e Londres - 1666 (cf. CHALINE e DUBOIS-MAURY, 1994).

Até esse momento, o risco era considerado uma categoria neutra, que produzia consequências tanto positivas quanto negativas. Na Renascença italiana, a definição e a percepção do risco adquirem um lugar destacado na sociedade europeia, bem como o seu sentido predominante na atualidade como algo negativo, a ser evitado e temido (VEYRET, 2007; MARANDOLA JR., 2004).

Na Modernidade, associados à atividade industrial e aos avanços técnico-científicos, Cutter (1993) enumerou algumas atividades que passaram a desencadear os chamados "technological risks" (riscos tecnológicos), e.g., o risco de ocorrência de câncer atribuído às linhas de transmissão de energia elétrica de alta voltagem; os riscos ligados ao uso de energia nuclear; os riscos atribuídos ao uso de armas químicas e biológicas (biohazard) nas guerras e conflitos .

Quanto aos primeiros estudos modernos sobre os riscos, Castro et al. (2005) atribuem ao clássico trabalho de Frank Knight, "Risk, uncertainty and profit", de 1921, o uso inicial dos termos "risco" e "incerteza", quando assumiram o papel de termos técnicos na literatura acadêmica.

\section{RISCOS E PERIGOS: uma tradição das ciências sociais e da Geografia}

Dada a histórica tradição geográfica de estudar o espaço com base em suas componentes naturais e sociais, foi na Geografia que surgiram os clássicos trabalhos sobre os "natural hazards". Por muito tempo, os geógrafos (principalmente geógrafos físicos) se mantiveram imbuídos estritamente da pesquisa dos aspectos físico-naturais, de seus processos, de suas cronologias e de sua mensuração.

Essa orientação se manteve até as décadas de 1950/60, quando houve um crescente despertar para o interesse das relações entre as atividades humanas e o ambiente. Um dos aspectos que suscitou o estreitamento entre estudos da natureza e estudos da sociedade na Geografia teve lugar nas pesquisas sobre os "acasos naturais", como destaca Gregory (1992) .

Ainda de acordo com Gregory, sempre houve frequentes alusões às relações homem-ambiente pelos geógrafos, mas estes optaram pela ignorância dos indicadores que se evidenciavam já na metade do século XIX e, por seu turno, a Geografia Física se desenvolveu praticamente de forma isolada e sem a consideração da ação humana no seu escopo teórico-metodológico.

Trabalhos pioneiros, como o de George Perkins Marsh, Man and Nature, de 1864, tiveram forte influência sobre as pesquisas posteriores, por demonstrar as formas como a Terra era vista e utilizada pelo homem.

Foi no final da década de 1950, entretanto, que uma das tendências de estudo da relação homem-ambiente e de inserção daquele nos estudos de Geografia Física surgiu, principalmente, no que concerne ao estudo dos "acasos terrestres" sob uma perspectiva socioeconômica (GREGORY, 1992).

À pesquisa do que se chama de "natural hazards" ou perigos naturais, atribui-se uma das tendências de inclusão das influências humanas nos estudos ambientais e na Geografia Física. A esse respeito, os trabalhos pioneiros do geógrafo norte-americano Gilbert F. White se tornaram referência nos estudos dos riscos e perigos naturais, e por isso merece uma atenção mais distintiva.

As pesquisas de White estavam pautadas na Ecologia Humana, subdisciplina desenvolvida no âmbito da Sociologia e da Geografia, na Universidade de Chicago, em meados do século XIX (MILETI, 1999). Reconhecido internacionalmente como o "pai" da pesquisa e da gestão dos "na- 
tural hazards", White baseou suas pesquisas na ideia de que os perigos naturais são o resultado da interação de forças naturais e sociais, e que os perigos e seus impactos podem ser reduzidos por ajustamentos individuais e coletivos (MILETI, 1999).

O próprio White exprimiu, em artigo produzido com Robert Kates e Ian Burton (WHITE et al., 2001), que a pesquisa sobre os "hazards" nasceu de uma demanda por estudos e análises com a finalidade de aplicação às situações práticas da realidade .

Apesar da colaboração de profissionais diversos na apresentação de soluções para este problema - diques, represas, revestimento de margens fluviais etc. - muitos geógrafos questionaram a ênfase dada a soluções pautadas estritamente em obras de engenharia e, a partir disso, esboçaram a possibilidade de soluções alternativas.

É o caso do artigo de White e colegas (1958) sobre as mudanças produzidas pela ocupação humana nas planícies inundáveis dos EUA, geridas de acordo com diretrizes administrativas da época, e que manifestaram um importante paradoxo: as perdas de todo o tipo ocasionadas pelas inundações estavam aumentando consideravelmente, no lugar de reduzir em função das obras de contenção (GARCÍA-TORNEL, 1984).

No final da década de 1960, a Comissão Homem Ambiente da União Geográfica Internacional (UGI) promoveu investigações sobre riscos naturais e suas consequências para o homem. As pesquisas realizadas entre 1968-72 foram posteriormente editadas por Gilbert White, então presidente da referida comissão e publicadas, em 1974 (WHITE, 1974), com o título "Natural hazards - local, national, global".

Também foi em meados da década de 1970 que foi se construindo uma tendência a uma abordagem mais conjuntiva dos "natural hazards". À época existia a abordagem dos "hazards", na Geografia, baseada na sua herança da Ecologia Humana, com ênfase nas relações entre os perigos naturais e as respostas sociais a estes e na redução das perdas (MILETI, 1999).

Já oriundo da Sociologia, surge uma abordagem cuja perspectiva era a da análise do comportamento coletivo e enfatizava a resposta aos desastres e à prevenção destes. Esta orientação teórica ficou conhecida como "disaster research", por sua postura em salientar o estudo dos desastres e pela formação de uma "escola" de pesquisa sobre esse tema .

A procura por abordagens mais integrativas começa a se materializar em 1972, quando as duas propostas anteriores, a geográfica, pautada na análise dos perigos ou "hazards"; e a sociológica, relacionada à escola de pesquisa dos desastres, foram agregadas com os diferentes panoramas da Climatologia, Engenharia, Economia, Direito, Planejamento, Psicologia, Meteorologia, Políticas Publicas, entre outras.

Em outro trabalho do grupo de White (BURTON, KATES e WHITE, 1978), os autores procuraram esboçar uma "história" dos avanços nas pesquisas sobre o tema dos perigos. Referido trabalho tornou-se umas das principais referências para pesquisadores e profissionais dos riscos e perigos, pois os autores concluíram que a frequência e a magnitude dos eventos catastróficos estavam aumentando, assim como suas perdas e custos, que recorrentemente recaem de forma diferenciada em relação a vários países.

Os autores também destacam a forte interdependência entre a dinâmica físico-natural da Terra e os processos de ocupação do território, este sendo o principal responsável pela crescida das incertezas e dos danos às populações, principalmente aquelas mais vulneráveis.

\section{UMA DISCUSSÃO DOS CONCEITOS DE RISCO, PERIGO E DESASTRE}

O risco é um constructo eminentemente social, ou seja, é uma percepção humana. Risco é a percepção de um indivíduo ou grupo de indivíduos da probabilidade de ocorrência de um evento potencialmente perigoso e causador de danos, cujas conseqüências são uma função da vulnerabilidade intrínseca desse indivíduo ou grupo. 
Observa-se que, em geral, confunde-se a noção de risco com a noção do próprio evento que causa ameaça ou perigo, o que dificulta a sua percepção e sua gestão. Assim, a noção de perigo, que é diferente da ideia de risco, tem relaçào com a possibilidade ou a própria ocorrência de um evento causador de prejuízo .

Outros dois conceitos que merecem esclarecimentos são desastre e catástrofe. De acordo com Quarantelli (1998), um desastre é um evento concentrado no tempo e no espaço, no qual uma comunidade experimenta severo perigo e destruição de seus serviços essenciais, acompanhado por dispersão humana, perdas materiais e ambientais, que frequentemente excedem a capacidade dessa comunidade em lidar com as consequências do desastre sem assistência externa. Já o conceito de catástrofe é semelhante ao de desastre. O que os diferencia é a escala ou a magnitude das consequências e, nesse caso, a catástrofe possui dimensões mais amplas, podendo ser quantificada quanto às perdas humanas, financeiras e ecológicas.

A segunda vertente do conceito de risco é a vulnerabilidade. Nesse contexto, o risco é função de duas categorias: $\mathrm{f}(\mathrm{R})=\mathrm{P} \times \mathrm{V}$, de onde Pé o próprio evento perigoso (perigo) ou a sua potencialidade de ocorrência, e V é a vulnerabilidade intrínseca de um indivíduo ou grupo de indivíduos. Outros conceitos imbuídos na ciência da vulnerabilidade, tais como resiliência, adaptação, insegurança, ajustamento, exposição e susceptibilidade, podem ser analisados na vasta bibliografia produzida sobre o tema.

De acordo com Veyret (2007), há diversos tipos de riscos, mas nem todos são tratados pela Geografia. Os riscos cuja percepção e gestão são acompanhadas de uma dimensão espacial, e por isso são abordados pela ciência geográfica, são classificados de acordo com os processos que os engendram. Dessa forma, a classificação dos riscos (que apresentam expressão espacial) pode ser assim sinteticamente estabelecida: riscos ambientais (riscos naturais e riscos naturais agravados pelo homem); riscos tecnológicos; riscos econômicos, geopolíticos e sociais; e outros tipos de riscos (ex.: riscos maiores - terremotos, tsunamis; riscos urbanos).

\section{A DÉCADA DE 1980 E A EMERGÊNCIA DA CIÊNCIA DA VULNERABILIDADE}

Ao analisar-se a distribuição socioespacial das vitimas mundiais de catástrofes naturais no período 1973-2002, tornam-se claras as diversas diferenças, no que diz respeito às consequências, entre os estados de pobreza dos países afetados. Os países mais pobres, ou seja, aqueles com Índice de Desenvolvimento Humano (IDH) inferior a 0,8 (= México) registraram neste período 96\% dos óbitos relativos a fenômenos naturais. Tal fato explicita que a pobreza é, indiscutivelmente, uma das causas mais profundas da vulnerabilidade (LEONE e VINET, 2006).

O crescimento das desigualdades sociais, da pobreza, da segregação socioespacial advinda com o trinômio capitalismo - industrialização - urbanização, em correlação com a consequente degradação do ambiente nas suas diversas facetas, fez surgir em meados dos anos 1980 uma abordagem teórico-metodológica que procurou enfocar os desastres (naturais ou tecnológicos) do ponto de vista não apenas de seus fatores físicos desencadeantes, mas com base no prisma das populações atingidas.

Até a década de 1970, a primazia das pesquisas sobre riscos se assentava sobre a análise dos perigos e ameaças naturais (os chamados "natural hazards"), cujos trabalhos procuravam respostas técnicas a situações ou problemas práticos do cotidiano (como visto anteriormente).

As investigações sobre os perigos naturais e as intervenções técnicas para a mitigação das consequências humanas e materiais (normalmente oriundas da engenharia de obras) tomou a forma de um paradigma chamado "Risk analysis" (Análise do risco), que passou a conduzir pesquisas e avaliações, dentro e fora da academia, com apoio em análises econômicas até consultorias de seguros (MARANDOLA JR. e HOGAN, 2004).

A premissa de que os desastres são consequências diretas de eventos ou ameaças físicas de tipos diferentes, base para o paradigma da análise do risco, e as diversas publicações sob essa égi- 
de, são definidas por Hewitt como abordagens "fisicalistas", ao confrontar com as abordagens que partem de uma visão dos desastres como uma problemática social, ou ainda como "problemas não resolvidos do desenvolvimento." (MASKREY, 1996).

Essa perspectiva de compreensão das catástrofes e desastres naturais provocados por fenômenos como terremotos, ciclones, furacões, erupções vulcânicas, deslizamentos de terra, avalanches, "tsunamis" e inundações passou a incorporar abordagens mais integradoras e holísticas no entendimento das complexas redes de relações social-natural-cultural-ambiental, que se configuram como a base da gênese dos desastres . É nesse contexto de inclusão das dimensões socioculturais na problemática ambiental que surge o conceito de vulnerabilidade. White et al. (2001) exprimem as tendências teórico-metodológicas da pesquisa sobre os perigos e desastres naturais e apontam a crescente atenção dada à abordagem da vulnerabilidade .

Nos últimos 50 anos, os pesquisadores da temática dos riscos e perigos focaram suas atenções na busca de respostas a uma série de questões fundamentais: como se dá a ocupação humana das zonas sob perigo ?; Como os indivíduos e as sociedades respondem aos perigos ambientais e que fatores influenciam suas escolhas de ajustamento (adaptação) ?; Como mitigar os riscos e os impactos dos perigos naturais ?. Na década de 1980, porém, outra questão foi posta à lista de perguntas anteriores: por que as sociedades estão se tornando mais vulneráveis aos perigos ambientais ? (CUTTER, 1996).

Definida grosso modo como o potencial de perda de um sistema (MITCHEL, 1989), a vulnerabilidade tornou-se um conceito essencial na abordagem dos riscos e perigos, e central para o desenvolvimento de estratégias de redução e mitigação das consequências dos desastres naturais, nas diversas escalas de análise (local, regional, nacional, global).

De acordo com Cutter (1996), a ciência da vulnerabilidade concentra-se em três áreas temáticas principais: as contradições e confusões no significado do termo; a sua medição; e as causas das resultantes espaciais associadas aos estudos de vulnerabilidade. A autora ainda acrescenta uma nova proposta ou um novo modelo conceitual de vulnerabilidade, definida como Os perigos do lugar (The hazards of place). Para White et al. (2001), corroborando a análise de Cutter, a vulnerabilidade se tornou, nas últimas décadas, um conceito central utilizado por diversos autores. Entre 12 livros sobre "natural hazards" analisados pelos autores, sete deles apresentavam o termo vulnerabilidade em seus respectivos índices, além de outras obras destacarem a definição do conceito e sua aplicabilidade (cf. quadro 1).

Dentre as ciências ambientais, a ciência da vulnerabilidade pode contribuir para o entendimento das circunstâncias que põem as pessoas sob risco e das condicionantes que reduzem a habilidade com que as pessoas e os lugares respondem às ameaças ambientais, ou seja, reduzem sua resiliência (CUTTER, 2003). A vulnerabilidade se tornou base para políticas de redução de riscos, perigos e desastres, como é o caso do IDNDR - International Decade for Natural Disaster Reduction, que é um importante programa de redução de perdas por riscos naturais, adotado em dezembro de 1989 pela Assembleia Geral das Nações Unidas, que proclamou os anos de 1990 como a década internacional de redução dos desastres naturais (MUNASINGHE e CLARK, 1995; SMITH, 2001).

Categorias de análise tais como risco (exposição), perigo, resiliência, susceptibilidade diferencial, recuperação/mitigação, também englobam em seus escopos teóricos o conceito de vulnerabilidade. Este também aparece da mesma forma, em pesquisas aplicadas aos estudos de desenvolvimento e pobreza (academicamente na Antropologia e na Sociologia), saúde pública, estudos de mudanças climáticas, Engenharia, Geografia, Ecologia Política e, principalmente, entre os pesquisadores dos riscos, perigos e desastres (BIRKMANN e WISNER, 2006).

Apesar da atual importância atribuída à ciência da vulnerabilidade, entretanto, o conceito ainda carece de melhor definição e de consenso em relação a ele, como constatam diversos autores (BOGARDI, 2004; CUTTER, 1996 e 2003; WHITE et al., 2001). As confusões e contradições no estabelecimento de uma definição consensual de vulnerabilidade implicam grandes dificuldades na 
operacionalização do conceito. $\mathrm{O}$ fato de haver um ramo de pesquisa tão profícuo sobre o debate do significado do termo pode servir de justificativa para a asserção anterior.

Quadro 1 - Seleção das publicações mais representativas dos anos 1990 sobre perigos naturais

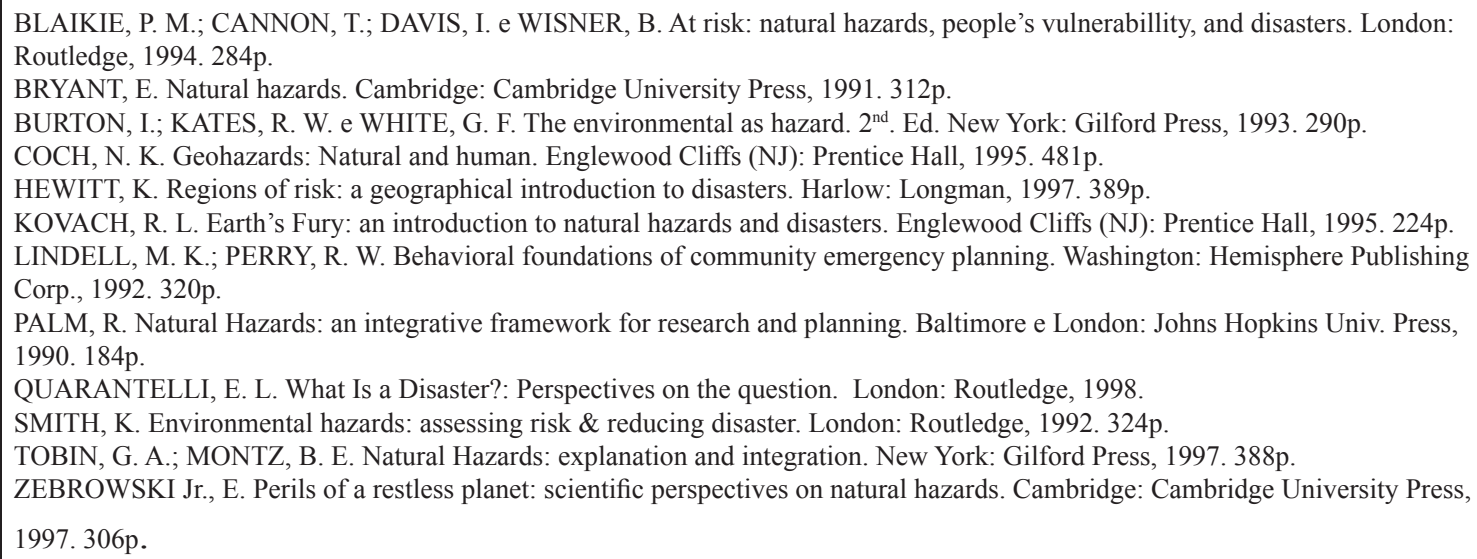

Fonte: White et al. (2001).

Nesse sentido, a definição mais ampla de vulnerabilidade como "potencial de perda" não articula de que perda se está descrevendo, e nem responde às seguintes questões postas por Cutter (1996): quem é/está vulnerável ?; Vulnerável a que processo ?; E em função de quais condições socioespaciais?

Com suporte nesses questionamentos, pode-se notar a multidimensionalidade do conceito, que permeia as várias dimensões que formam a realidade - cultural, social, econômica, ecológica, tecnológica, ambiental, psicológica, entre outras. Cutter (1996) relacionou 18 definições de vulnerabilidade, demonstrando essa multidimensionalidade, mas também uma evolução do conceito ao longo do tempo, como Hogan e Marandola Jr. (2006) exprimem.

A falta de consenso na definição do conceito de vulnerabilidade advém tanto da dificuldade em se apreender a multidimensionalidade da realidade analisada quanto da diversidade de orientações epistemológicas (Ecologia política, Ecologia humana, Ciências Físicas, Análise espacial, etc.) e, conseqüentemente, das práticas metodológicas (ou seja, da operacionalização do conceito).

Também nesse sentido, há considerável variação na escolha dos perigos e ameaças a serem analisados (seca, terremotos, inundações, fome, violência, entre outros), cada um com dimensões espaciotemporais específicas, além da escolha da região a ser estudada - países desenvolvidos ou em desenvolvimento, para citar um exemplo.

E Cutter (1996, p. 530) conclui: "the result is a confused lexicon of meanings and approaches to understanding vulnerability to environmental hazards". Assim, haveria maior evolução operacional do conceito se na sua aplicação fosse possível responder como e por que lugares e pessoas são vulneráveis aos perigos ambientais .

O conceito de vulnerabilidade, assim como o de risco, indica uma situação ou estado futuro. Nesse caso, a definição de Blaikie et al. (1994) parece oportuna, já que, para os autores, estar vulnerável significa compreender as características de uma pessoa ou grupo no que concerne à sua capacidade para antecipar, sobreviver, resistir e recuperar-se do impacto de uma ameaça ou perigo natural.

Os autores consideram o conceito como uma combinação de fatores que determinam o grau com que a vida e a subsistência de alguém são postas em risco por um evento distinto e identificável na natureza e/ou na sociedade.

A esse respeito, Smith (2001) entende que, para os mais vulneráveis, o acesso a recursos (e.g., 
a obtenção de um meio de vida seguro ou a formas de recuperação dos desastres) e a informação, e a disponibilidade de uma rede social que mobilize apoio e ajuda na comunidade, são fatores de enorme relevância para lidar com as consequências dos desastres.

Além disso, fatores como pobreza, idade, gênero, etnia, incapacidade, classe ou status social, casta, são características que podem indicar se determinados grupos da sociedade são mais propensos do que outros ao dano, à perda e ao sofrimento no contexto das diferentes ameaças .

Com efeito, em torno de $25 \%$ da população mundial vive em áreas de risco de perigos naturais. Anderson (1995) indica que a maior parte desse contingente populacional se encontra em países em desenvolvimento, onde a vulnerabilidade se forma da pobreza, da segregação/discriminação e da carência de representação política, que impedem o processo de desenvolvimento.

Pobreza, ocupação de lugares sujeitos a perigos naturais e/ou tecnológicos, concentração populacional nas cidades, impactos econômicos dos desastres, carências na infraestrutura e serviços, características sociais (citadas anteriormente - gênero, idade, classe etc.), degradação ambiental, corrupção, decisões políticas, carência de programas sociais, entre outros, - nos países em desenvolvimento, esses amplos problemas combinados criam as condições para o mais elevado grau de vulnerabilidade. Assim, pode-se inferir que a escala do impacto de um desastre é uma função da vulnerabilidade humana e da magnitude física do evento perigoso (cf. SMITH, 2001).

Bogardi (2004) fala em milhões de migrantes que se dirigem a cada ano para as cidades, em função da insustentabilidade e do declínio das comunidades rurais, e complementa: "It is widely expected that in 2025 two-third of humanity, thus 5 billion people by them, would live in cities." (P. 362). Para o autor, o crescimento urbano implacável e não planejado cria os próprios problemas: serviços municipais sobrecarregados e ocupação de terras marginais inseguras (vertentes instáveis e áreas propensas a inundações, na sua maioria).

Assim, parcela majoritária dos principais aglomerados urbanos está localizada nas zonas costeiras, em deltas e ao longo dos rios. O aumento da concentração de pessoas nesses ambientes amplifica consideravelmente o número de pessoas susceptíveis a serem expostas às consequências de eventos extremos, como tempestades, furacões, tufões, e eventos ulteriores, como movimentos de terra, tsunamis, inundações, entre outros (BOGARDI, 2004).

Embora o conceito de vulnerabilidade seja uma "chave" para a compreensão da segurança humana, há ainda uma forte incerteza quanto ao potencial de seu uso como categoria de análise dos riscos e perigos. Há fortes desafios para esse intuito, e um deles é o de ordem escalar. Enquanto as consequências ligadas aos problemas anteriormente descritos são de ordem global, a ocorrência de eventos extremos e suas superposições em relação à degradação ambiental são fenômenos de ordem local ou regional. Por isso, entender a sequência lógica e a natureza "estocástica" da cadeia "perigos-riscos-vulnerabilidade" é de suma importância, de acordo ainda com Bogardi (2004).

\section{A OPERACIONALIZAÇÃO DO CONCEITO DE VULNERABILIDADE}

Apesar de haver, porém, um relativo consenso no que concerne aos conceitos de perigo ("hazard") e de risco ("risk"), há ainda algumas incertezas quanto à amplitude e à aplicabilidade do conceito de vulnerabilidade ("vulnerability"). Como visto em Cutter (1996), há uma múltipla gama de definições para o conceito, de acordo com a sua revisão realizada em meados dos anos 1990.

Dos anos 2000 em diante, a procura do consenso conceitual persiste, e uma das definições mais simples e compreensíveis é reportada a Wisner (2002, apud BOGARDI, 2004, p. 362): "likelihood of injury, death, loss, disruption of livelihood or other harm in an extreme event, and/or unusual difficulties in recovering from such effects".

Na revisão global das iniciativas de redução de desastres (revisão do IDNDR), o International Strategy for Disaster Reduction (ISDR) define vulnerabilidade como "a set of conditions and processes resulting from physical, social, economical, and environmental factors, which increase the 
susceptibility of a community to the impact of hazards" (ISDR, 2002 apud BOGARDI, 2004, p. 362).

Da mesma forma, o relatório sobre redução de risco de desastre da UNDP (United Nations Development Programme) sublinha a conotação social da vulnerabilidade e a define como "a human condition or process resulting from physical, social, economic and environmental factors, which determine the likelihood and scale of damage from the impact of a given hazard." (UNDP, 2004 apud BOGARDI, 2004, p. 362).

Já Bohle expõe a natureza multifacetada da vulnerabilidade quando a concebe a sob dois prismas: um "externo" (ambiental) e outro "interno" (humano), cobrindo uma extensa gama de possíveis danos e consequências, o que implica um período relativamente longo, excedendo certamente o tempo de ocorrência do evento danoso (Bohle, 2002). Para Bogardi (IBID.), a interpretação de Bohle relaciona fortemente a vulnerabilidade à noção de resiliência, que significa, no caso específico dos desastres, a habilidade de retornar a um estado similar à condição anterior ao desastre.

Já Pelling (2003), tratando da vulnerabilidade humana (individual), divide a vulnerabilidade aos perigos naturais em três componentes: exposição ("exposure"), resistência ("resistenace") e resiliência ("resilience") (Pelling, 2003 apud Klein et al., 2003). Seguindo a proposta de Blaikie et al. (1994), Pelling descreve a resiliência aos perigos naturais como a habilidade de um ator em "lidar com" ("to cope with" - em inglês, "faire face" - em francês) ou adaptar-se a ocorrência de um evento perigoso .

Em Dauphiné (2005), a noção de vulnerabilidade se apresenta como a segunda vertente do conceito de risco (a outra categoria é a concepção de perigo ou "aléa" em francês, ou "hazard" em inglês). Em função das numerosas definições que o conceito de vulnerabilidade possui, o autor agrupa os conjuntos de definições em duas grandes categorias, uma dita analítica e outra dita sintética. A definição analítica considera a vulnerabilidade, num sentido amplo, como a expressão do nível de consequências previsíveis de um fenômeno natural sobre os recursos ameaçados, estes representados pelo homem, seus bens e o ambiente em que vive. Essa abordagem é tida como analítica, já que os recursos vulneráveis podem ser decompostos, por exemplo, no caso de bens econômicos, quando é possível calcular os danos para a agricultura, a indústria e os serviços.

Após algumas décadas, no entanto, essa definição de vulnerabilidade se mostrou restritiva e oposta a uma abordagem que entenderia a vulnerabilidade das sociedades com base em suas capacidades de resposta às crises potenciais (D'ERCOLE, 1994). Essa concepção de vulnerabilidade (sintética) intenta traduzir a fragilidade de um sistema no seu conjunto e, de forma indireta, demonstrar a sua capacidade de superar a crise provocada por um perigo potencial (aléa). Assim, quanto mais um sistema é apto a se restabelecer após uma catástrofe, menos ele é considerado vulnerável, o que remete novamente às noções de resistência e resiliência.

Ao estimar o nível de disponibilidade operacional dos métodos de avaliação das vulnerabilidades às ameaças naturais, Leone e Vinet (2006) concebem duas formas de operacionalização do conceito de vulnerabilidade, quais sejam: abordagens setoriais e abordagens globais e sistêmicas.

Tratando das abordagens setoriais (semelhante ao conceito analítico de vulnerabilidade de Dauphiné, 2005), é possível distinguir ou decompor a vulnerabilidade ao "infinito" em numerosos tipos, mas, para efeito dos estudos dos fenômenos naturais, tem-se o quadro 2.

No que diz respeito à abordagem global ou sistêmica (sintética, para Dauphiné, 2005), não são poucos os autores que descrevem as dificuldades para operacionalizar abordagens conjuntivas, holísticas, e inter, multi e pluridisciplinares da vulnerabilidade. A impossibilidade de se estabelecer uma vulnerabilidade total obriga os pesquisadores dessa ciência à busca de formas relativas de mensuração .

O trabalho de Chardon (1994) sobre a vulnerabilidade da cidade de Manizales (Colômbia) diante de um conjunto de riscos naturais (inundações, sismos, escorregamentos de terra), é um exemplo da procura de operacionalização metodológica mais global e sistêmica, mesmo apresentando, a princípio, estudos setoriais: o uso de indicadores classificados em duas categorias principais 
- vulnerabilidade física e socioeconômica - tratou da elaboração de uma hierarquização espacial da vulnerabilidade e sua respectiva cartografia.

Quadro 2 - Tipos de vulnerabilidade aplicados aos estudos dos fenômenos naturais

\begin{tabular}{|l|l|}
\hline \multicolumn{1}{|c|}{ TIPO DE VULNERABILIDADE } & \multicolumn{1}{c|}{ CARACTERÍSTICAS } \\
\hline Vulnerabilidade física (ou estrutural, ou corporal) & $\begin{array}{l}\text { Concentram-se na análise das construções, das redes de infraestrutura e do } \\
\text { potencial de perdas humanas. }\end{array}$ \\
\hline Vulnerabilidade humana ou social & $\begin{array}{l}\text { Avalia os retornos de experiência sobre as capacidades de resposta, adap- } \\
\text { tações, comportamentos e suas consequências socioeconômicas e terri- } \\
\text { toriais. Acrescenta-se ainda a percepção das ameaças ou da memória do } \\
\text { risco, o conhecimento dos meios de proteção, os tipos de comportamentos } \\
\text { potenciais. }\end{array}$ \\
\hline Vulnerabilidade institucional & $\begin{array}{l}\text { Trata da capacidade de resposta das instituições diante da crise; funciona } \\
\text { como fator indireto da vulnerabilidade social. }\end{array}$ \\
\hline Vulnerabilidade ambiental e patrimonial & $\begin{array}{l}\text { Analisa os danos sobre os componentes ambientais - vegetação, solos, } \\
\text { recursos hídricos, fauna, e aspectos culturais provocados por fenômenos } \\
\text { naturais. }\end{array}$ \\
\hline Vulnerabilidade funcional e econômica & $\begin{array}{l}\text { Avalia as disfunções no que tange às atividades econômicas, rupturas nas } \\
\text { redes de comunicação e transporte, entre outros. }\end{array}$ \\
\hline
\end{tabular}

Fonte: elaborado com apoio em Leone e Vinet (2006)

A esse respeito, D’Ercole (1994) adverte para a dificuldade de apreender a vulnerabilidade contida na pluralidade de variáveis que a compõem no âmbito dos espaços urbanos, notadamente aqueles nos países em desenvolvimento.

A l'approche classique de la vulnérabilité qui mesure un endommagement potentiel des biens et des personnes et ses répercussions sur l'environnement économique, semble s'opposer celle qui considère la vulnérabilité des sociétés à travers leur capacité de réponse à des crises potentielles. Cette capacité est elle-même liée à un ensemble de facteurs structurels et contingents qui peuvent être analysés séparément, mais dont les interrelations s'avèrent complexes. C'est pourquoi, toute analyse de vulnérabilité, surtout lorsqu'il s'agit du monde urbain qui tend à multiplier et diversifier les facteurs de vulnérabilité, peut difficilement s'affranchir d'une démarche systémique. (D'ERCOLE, 1994, P. 94).

Há exemplos de trabalhos que evocam o uso da análise de sistemas na avaliação da vulnerabilidade nos espaços urbanos. Leone e Vinet (2006) citam os trabalhos do IRD - Institut de Recherche pour le Développement, no aglomerado metropolitano de Quito (Equador), no quadro de elaboração de um sistema de informação e riscos (principalmente vulcânicos); a vulnerabilidade dos sistemas urbanos diante dos riscos naturais, tecnológicos e sociais foram objeto de outros estudos ditos sistêmicos, como é o caso do trabalho de Chardon (1994) anteriormente citado.

Leone e Vinet (2006) também abordam outros desafios ligados à operacionalização do conceito de vulnerabilidade, no que diz respeito à escolha dos territórios estudados e seus contextos socioeconômicos (e.g., países em desenvolvimento); às escalas espaciais de análise (e.g. escala local - urbana, bacia do risco; escala regional - vulcão, bacia hidrográfica, região); as ferramentas de avaliação utilizados (e.g., SIG, questionários, cartografia); e as disciplinas e profissionais mobilizados (e.g., arquitetos, geólogos, engenheiros, sismólogos, vulcanólogos, geomorfólogos e, principalmente, os geógrafos).

Quanto aos geógrafos, os autores destacam a importância destes profissionais pela sua formação pluridisciplinar e pela visão de interface sobre os fenômenos perigosos e os recursos vulneráveis, administrando abordagens espaciais e ferramentas cartográficas, o que fez com que se percebesse uma tendência ao desenvolvimento de uma nova subdisciplina: a Geografia dos Riscos Naturais. 


\section{MENSURAÇÃO DA VULNERABILIDADE: desafios e oportunidades}

Um dos desafios mais importantes na operacionalização da vulnerabilidade, no entanto, diz respeito a sua mensuração. Após as grandes catástrofes naturais recentes - o tsunami no oceano Índico em 2004, e o furacão Katrina em 2005 - houve uma forte exposição das vulnerabilidades das diversas sociedades ao impacto dos perigos naturais.

A partir de então, o desenvolvimento de metodologias de medição da vulnerabilidade se tornaram prerrequisito para a promoção da redução dos riscos e a preparação contra os desastres. Durante a WCDR - World Conference on Disaster Reduction, ocorrida em Kobe, no Japão, em 2005, identificou-se o fato de que o desenvolvimento de indicadores para a medida da vulnerabilidade e do risco, bem como a sua redução, se tornaram fortes desafios para o futuro (BOGARDI, 2006).

In this context the term 'measuring vulnerability' does not solely encompass quantitative approaches. It also seeks to discuss and develop all types of methods able to translate the abstract concept of vulnerability into practical tools, classifications and comparative judgments to be applied in the field. (BOGARDI, 2006, p. 05).

Já de acordo com Birkmann (2007), em contraste com o relevante desenvolvimento de mecanismos de resposta aos desastres no âmbito da comunidade internacional, o desenvolvimento de uma metodologia comum para identificar e mensurar os riscos e as vulnerabilidades aos desastres para a definição de formas da gestão dos riscos/desastres e de prioridades nessa gestão, ainda não foram suficientemente desenvolvidos.

Nesse sentido, para reforçar o processo de mensuração dos riscos/vulnerabilidades, é preciso realçar o conhecimento sobre: os mais vulneráveis; os espaços expostos a risco; e os fatores que influenciam e produzem vulnerabilidade/risco (BIRKMANN, 2007).

No trabalho de Birkmann (2007), o objetivo principal foi realizar uma revisão de quatro pesquisas que tiveram entre seus objetivos a medição de riscos e vulnerabilidades, mediante utilização de indicadores, aplicados em diversas escalas espaciais. Dessa forma, todas as abordagens analisadas consideraram que o risco de desastre é produto de exposição aos perigos; frequência e magnitude do perigo; e vulnerabilidade.

Num relatório semelhante à pesquisa anterior, Birkmann e Wisner (2006) enfatizaram que os estudos de caso analisados revelaram que a medição ou mensuração da vulnerabilidade requer diferentes abordagens, dependendo da ameaça em questão (perigo) e do contexto socioeconômico e cultural do espaço analisado. O trabalho de Birkmann e Wisner (2006) resultou dos debates ocorridos na WCDR, reunião promovida pelo Expert Working Group on Measuring Vulnerability da United Nations University (UNU-EWG), e de acordo com a síntese realizada após a apresentação dos diversos métodos aplicados a estudos de caso, há grandes áreas temáticas emergentes ligadas à mensuração da vulnerabilidade, tais como temáticas sociais, econômicas, ambientais e institucionais.

Dentre as pesquisas analisadas, os autores destacaram o trabalho de Birkmann e colegas sobre a produção de um modelo dito de aplicação "rápida" e multidimensional de análise da vulnerabilidade do Sri Lanka à ocorrência de tsunamis no oceano Índico. A pesquisa baseou-se no modelo conceitual BBC e utilizou quatro metodologias para identificar e medir vulnerabilidades, capacidades de resposta e ferramentas apropriadas de intervenção .

No Brasil, é crescente o número de pesquisas que tratam da hierarquização espacial e da elaboração de índices de vulnerabilidade, procurando avaliar as desigualdades sociais e ambientais e reduzir os riscos atrelados a eventos naturais e tecnológicos. Cita-se como exemplo o Índice Paulista de Vulnerabilidade Social, elaborado pela Fundação SEADE (Fundação Sistema Estadual de Análise de Dados) do Estado de São Paulo (SEADE, 2008). Outros exemplos brasileiros que valem referência são os de Deschamps (2004) e Dantas e Costa (2009). 
Já Almeida (2010) desenvolveu, em tese de doutoramento, metodologia de mensuração de vulnerabilidades socioambientais de rios urbanos no Brasil, com aplicação à bacia hidrográfica do rio Maranguapinho, localizado na Região Metropolitana de Fortaleza. Os resultados da pesquisa indicam que há tendência, na América Latina, e sobretudo no Brasil, de coincidência entre os espaços mais expostos aos processos naturais perigosos - inundações, no caso específico - e os espaços ocupados por população socialmente vulnerável.

A integração ou sobreposição dos mapas produzidos com arrimo no Índice de Vulnerabilidade Social - IVS e no Índice de Vulnerabilidade Físico-Espacial às Inundações - IVFI, possibilitou a identificação e localização dos espaços onde ocorre coincidência de riscos e vulnerabilidades - sociais e ambientais - resultando num Índice de Vulnerabilidade Socioambiental - IVSA para bacia hidrográfica do rio Maranguapinho, localizada na Região Metropolitana de Fortaleza, representado graficamente pelo Mapa de Vulnerabilidade Socioambiental (cf. ALMEIDA, 2010).

Inicialmente, definiram-se a legenda do mapa e os respectivos grupos de Vulnerabilidade Socioambiental mediante o cruzamento dos grupos de vulnerabilidade dos índices produzidos anteriormente (figura 2). Propôs-se o cruzamento entre os grupos de vulnerabilidade (social e físico-espacial) com suporte em suas proporcionalidades, ou seja, grupos com hierarquias semelhantes (e.g., vulnerabilidade social alta/vulnerabilidade físico-espacial alta).

A sobreposição dos mapas seguiu a legenda elaborada anteriormente e integrou os setores censitários do IVS com as áreas de extensão espacial das inundações do IVFI que apresentavam índices de vulnerabilidade proporcionais, formando assim grupos homogêneos de vulnerabilidade socioambiental e possibilitando a identificação e localização de espaços em que ocorre coincidência de vulnerabilidades sociais e ambientais na bacia hidrográfica do rio Maranguapinho (figuras 2, 3 e 4).

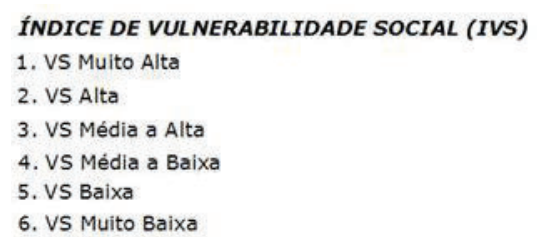

\section{INDICE DE VULNERABILIDADE FISICO-ESPACIAL} ÀS INUNDAÇÕES (IVFI - TEMPOS DE RETORNO)

A. TR $\leq 2$ anOS - VFE MUITO ALTA

B. $T R \leq 20$ anOs - VFE ALTA

C. $T R \leq 100$ anOs - VFE MÉDIA A BAIXA

D. TR $>100$ anOS - VFE BAIXA A MUITO BAIXA

INDICE DE VULNERABILIDADE SÓCIOAMBIENTAL - IVSA (IVS X IVFI)

\begin{tabular}{|c|c|c|c|c|}
\hline IVS IVA & A & B & C & D \\
\hline 1 & & & & \\
\hline 2 & & & & \\
\hline 3 & & & & \\
\hline 4 & & & & \\
\hline 5 & & & & \\
\hline 6 & & & & \\
\hline
\end{tabular}

\begin{tabular}{|l|l|}
\hline \multicolumn{2}{|c|}{ IVSA (IVS X IVFI) } \\
\hline & VSA Muito Alta \\
& VSA Alta \\
VSA Média a Alta \\
VSA Média a Baixa \\
VSA Baixa \\
VSA Muito Baixa \\
\hline
\end{tabular}

Figura 2 - Metodologia de elaboração da legenda do Mapa de Vulnerabilidade Socioambiental - IVSA da bacia hidrográfica do rio Maranguapinho Fonte: Almeida (2010). 


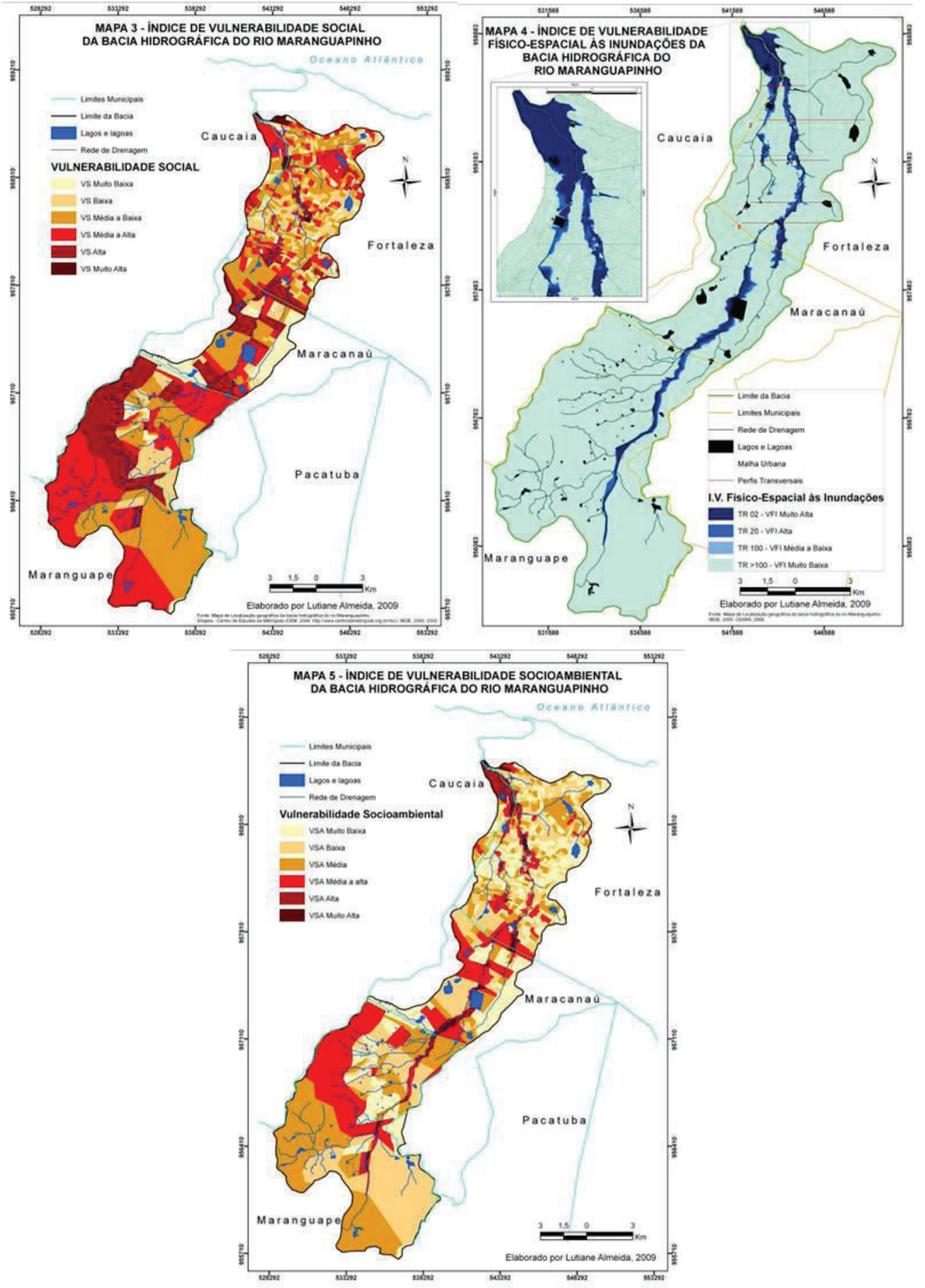

Figura 2, 3 e 4 - Mapas de Vulnerabilidade Social, de Vulnerabilidade Físico-Espacial às inundações e Vulnerabilidade Socioambiental da bacia hidrográfica do rio Maranguapinho. Fonte: Almeida (2010).

\section{CONSIDERAÇÕES FINAIS}

Risco e vulnerabilidade são conceitos que estão na moda. Foram inseridos nas mais variadas agendas governamentais e/ou não-governamentais em todo o Planeta, vide a International Decade for Natural Disaster Reduction - IDNDR, nos anos 1990, que culminou na criação da International Strategy for Disaster Reduction - ISDR, e mais recentemente, o $4^{\circ}$ relatório do Intergovernmental Panel on Climate Change - IPCC. 
No Brasil, os conceitos de risco e vulnerabilidade ainda carecem de maior discussão e inserção no escopo teórico e metodológico da Geografia. Nesse caso, urge pensar numa ciência dos riscos e vulnerabilidades na Geografia, criando mecanismos de divulgação das principais propostas teóricas que conduzem esses conceitos-chave, notadamente em língua portuguesa, dada a carência que se esboça, se comparada à produção acadêmica sobre o tema em língua inglesa, francesa ou espanhola.

Urge, também, pensar o desafio da operacionalização do conceito de vulnerabilidade. Essa difícil tarefa pode auxiliar a identificação de espaços prioritários para investimentos que possam melhorar as condições de resiliência das comunidades que se apresentam mais propensas aos perigos naturais por suas vulnerabilidades sociais. Assim, o emprego desse conceito e a sua operacionalização podem contribuir para a tomada de decisões que possibilitem a redução dos riscos de desastres naturais.

Mesmo sendo a definição, compreensão e operacionalização problemáticas, em função da complexidade e multidimensionalidade, pode-se afiançar que o conceito de vulnerabilidade pode auxiliar a identificação das características sócioespaciais de determinadas comunidades (e indivíduos) que influenciam nas suas capacidades de resposta e recuperação diante dos perigos naturais.

\section{REFERÊNCIA BIBLIOGRÁFICAS}

ALLARD, Paul. Éléments pour une problématique de l'histoire du risque. Du risque accepté au risque maîtrisé. Représentations et gestion du risque d'inondation en Camargue, XVIIIe-XIXe siècles. In : Ruralia, 2000-06, [on line]. Em: http://ruralia.revues.org/document152.html. Acesso em 16/08/2008.

ALMEIDA, L. Q. de. Vulnerabilidades socioambientais de rios urbanos. Bacia hidrográfica do rio Maranguapinho. Região Metropolitana de Fortaleza, Ceará. Pós-Graduação em Geografia. Instituto de Geociências e Ciências Exatas/Unesp, Rio Claro, Tese de Doutorado, 2010. 278p. Em:

http://www.athena.biblioteca.unesp.br/exlibris/bd/brc/33004137004P0/2010/almeida_lq_dr_rcla.pdf

ANDERSON, Mary. B. Vulnerability to disaster and sustainable development: a general framework for assessing vulnerability. In : MUNASINGHE, Mohan.; CLARKE, Caroline. Disaster prevention for sustainable development: economic and policy issues. Washington (DC): IDNDR/The World Bank, 1995, p. 41-59.

ANEAS DE CASTRO, Susana. D. Riesgos y peligros: una visión desde lá Geografía. Scripta Nova: Revista Electrónica de Geografía y Ciencias Sociales. Barcelona, n.60, 15 de mar. 2000. Em: http://www.ub.es/ geocrit/sn-60.htm .

ANTOINE, Jean-Marc. et al. Les mots des risques naturels. Toulouse (FR): Presses Universitaires du Mirail, 2008.

BECK, Ulrich. La sociedad del riesgo. Barcelona: Paidós, 1998.

BERNSTEIN, Peter. L. Against the Gods: the remarkable story of risk. Nova Jersey: John Wiley and Sons, 1998.

BIRKMANN, Jörn. Risk and vulnerability indicators at different scales: applicability, usefulness and policy implications. Environmental hazards. v. 7, p. 20-31, 2007.

BIRKMANN, Jörn.; WISNER, Ben. Measuring the Un-Measurable: The Challenge of Vulnerability. Bonn (Alemanha): UNU-EHS, 2006.

BLAIKIE, Piers. M.; CANNON, Terry.; DAVIS, Ian. ; WISNER, Ben. At risk: natural hazards, people's vulnerabillity, and disasters. London: Routledge, 1994. 284p.

BOGARDI, Janos. J. Foreword. In: BIRKMANN, J.; WISNER, B. Measuring the Un-Measurable: The Challenge of Vulnerability. Bonn (Alemanha): UNU-EHS, 2006.

BOGARDI, Janos. J. Hazards, risks and vulnerabilities in a changing environment: the unexpected onslaught on human security? Global Environmental hazards. v. 14, n. 4, dez. p. 361-365, 2004.

BOHLE, Hans. G. Land degradation and human security. In : PLATE, E. (ed.) Environment and human security. Contributions to a workshop in Bonn. Bonn, 2002.

BURTON, Ian.; KATES, Robert. W.; WHITE, Gilbert. F. The Environment as Hazard. New York: Oxford University Press, 1978. 
CASTRO, Cleber. M.; PEIXOTO, Maria. N. O.; RIO, Gisela. A. P. Riscos Ambientais e Geografia: Conceituações, Abordagens e Escalas. In: Anuário do Instituto de Geociências - UFRJ. Rio de Janeiro: UFRJ, Vol. 28-2, 2005 p. 11-30.

CERRI, Leandro. E. S.; AMARAL, C. P. Riscos Geológicos. In: OLIVEIRA, A. M. S.; BRITO, S. N. A. (eds.) Geologia de Engenharia. São Paulo: ABGE, 1998.

CHALINE, Claude.; DUBOIS-MAURY, Jocelyne. La ville et ses dangers: prévention et gestion des risques naturels, sociaux et technologique. Paris: Masson, 1994.

CHARDON, Anne-Catherine. Etude intégrée de la vulnérabilité de la ville de Manizales (Colombie) aux risques naturels. Revue de Géographie Alpine, 1994, V. 82, n. 4, p. 97 - 111.

CUTTER, Susan. L. Living with risk: the geography of technological hazards. Londres: Arnold, 1993.

CUTTER, Susan. L. The vulnerability of science and the science of vulnerability. Annals of the Association of American Geographers, v.93, n.1, p.1-12, 2003.

CUTTER, Susan. L. Vulnerability to environmental hazards. Progress in Human Geography, v.20, n.4, p.529-539, 1996.

DANTAS, Eustógio.; COSTA, Maria. C. L. (Orgs.) Vulnerabilidades socioambiental na Região Metropolitana de Fortaleza. Fortaleza: Edições UFC, 2009.

DAUPHINÉ, André. Risques et catastrophes. Observer, spatialiser, comprendre, gérer. Paris: Armand Colin, 2005.

D’ERCOLE, Robert. Les vulnérabilités des sociétés et des espaces urbanisés: concepts, typologie, modes d'analyse. Revue de Géographie Alpine, 1994, V. 82, n. 4, p. 87 - 96.

DESCHAMPS, Marley. V. Vulnerabilidade socioambiental na região metropolitana de Curitiba/PR. Curitiba, Tese (Doutorado em Meio Ambiente e Desenvolvimento) - Universidade Federal do Paraná. 2004. 155p.

DUCLOS, Denis. L'homme face au risque technique. Paris: l'Harmattan, 1991.

FOUCHER, Michel. Esquisse d'une géographie humaine des risques naturels. In: Terres a hauts risques. Hérodote. n. 24, jan.-abr., 1982.

GARCÍA-TORNEL, Francisco. C. La geografía de los riesgos. Geocrítica: Cuadernos Críticos de Geografía Humana, Barcelona, ano IX, n.54, nov. 1984. Em: http://www.ub.es/geocrit/geo54.htm .

GIDDENS, Anthony. Modernidade e identidade. Rio de Janeiro: Jorge Zahar Ed., 2002.

GREGORY, Kennett. J. A natureza da Geografia Física. (trad. Eduardo de A. Navarro). Rio de Janeiro: Bertrand Brasil, 1992. 367p.

HOGAN, Daniel. J.; MARANDOLA JR., Eduardo Para uma conceituação interdisciplinar da vulnerabilidade. In: CUNHA, J. M. P. Novas metrópoles paulistas: população, vulnerabilidade e segregação. Campinas (SP): NEPO/UNICAMP, 2006.

ISDR/UN/WMO. Terminology on Disaster Risk Reduction. On line. IDSR, 2009. Acesso em 27 fev 2009. http://www.unisdr.org/eng/library/lib-terminology-eng.htm

JONES, David. Environmental hazards in the 1990s: problems, paradigms and prospects. Geography, v.78, n.2, p.161-165, 1993.

KLEIN, Richard. J. T. et al. Resilience to natural hazards: how useful is this concept ? Environmental hazards. v. 5, p. 35-45. 2004.

LEONE, Frederic.; VINET, Freddy. La vulnerabilité, un concept fondamental au coeur des méthodes d'évaluation des risques naturels. In : LEONE, F.; VINET, F. La vulnerabilité des sociétés et des territoires face aux menaces naturelles: analyses géographiques. Montpellier : Université Paul Valery, Collection Géorisques n. 1, 2006, 144p.

MARANDOLA JR., Eduardo. Uma ontologia geográfica dos riscos: duas escalas, três dimensões. Geografia, Rio Claro, v.29, n.3, p.315-338, set./dez. 2004.

MARANDOLA Jr., Eduardo.; HOGAN, Daniel. J. O risco em perspectiva: tendências e abordagens. Geosul, Florianópolis, v. 19, n. 38, p. 25-58, jul./dez. 2004.

MASKREY, A. Prefacio a la edición en español. In: BLAIKIE, P. M.; CANNON, T.; DAVIS, Ian. e WISNER, Ben. Vulnerabilidad: el entorno social, politico y económico de los desastres. Ciudad de Panamá: 
LA RED, 1996.

MILETI, Dennis. S. Disasters by design: a reassessment of natural hazards in the United States. Washington: Joseph Henry Press, 1999.

MITCHELL, James. K. Hazards research. In: GAILE, G. L.; WILLMOTT, C. J. Geography in America. Columbus, OH: Merrill, 410-424, 1989.

MUNASINGHE, Mohan. ; CLARKE, Caroline. Disaster prevention for sustainable development: economic and policy issues. Washington (DC) : IDNDR/The World Bank, 1995.

PELLING, Mark. The vulnerability of cities: natural disaster and social resilience. London: Earthscan, 2003.

QUARANTELLI, Enrico. L. (ed.) What is a disaster ? Londres e Nova York: Routledge, 1998.

SEADE. Índice Paulista de Vulnerabilidade Social. Espaços e Dimensões da pobreza nos Municípios do Estado de São Paulo. Disponível em www.seade.gov.br/produtos/ipus/pdf/oipvs/pdf . Acesso 12/09/2008.

SMITH, Keith. Environmental hazards: assessing risk and reducing disaster. 3a. ed. London: Routledge, 2001. 392p.

TOSINI, Maria. F. C. Risco ambiental para as instituições financeiras. São Paulo: Annablume, 2006.

UNFPA. Situação da populacao mundial 2007. Desencadeando o potencial do crescimento urbano. [on line]. Acesso em http://www.unfpa.org.br/relatorio2007/swp2007_por.pdf .

VEYRET, Yvette. Os riscos: o homem como agressor e vítima do meio ambiente. São Paulo: Contexto, 2007. WHITE, Gilbert. F. et al. Changes in urban occupance of flood plains in the United States. University of Chicago, Department of Geography. Research Paper 57, Chicago, 1958, 235 p.

WHITE, Gilbert. F.; HAAS, J. Eugene. Assessment of research on natural hazards. Cambridge: MIT Press, 1975.

WHITE, Gilbert. F.; KATES, Robert. W.; BURTON, Ian. Knowing better and losing even more: the use of knowledge in hazards management. Environmental hazards. v. 3, n. 3-4, set./dez., p. 81-92, 2001.

Trabalho enviado em setembro de 2011

Trabalho aceito em outubro de 2011 